\title{
Primeiro episódio da esquizofrenia e assistência de enfermagem
}

\author{
FIRST EPISODE OF SCHIZOPHRENIAAND NURSING CARE
}

PRIMER EPISODIO DE LAESQUIZOFRENIAY ASISTENCIA DE ENFERMERÍA

\author{
Bianca Cristina Ciccone Giacon', Sueli Aparecida Frari Galera²
}

\begin{abstract}
1 Aluna do terceiro semestre de graduação do curso de Enfermagem da Escola de Enfermagem de Ribeirão Preto da Universidade de São Paulo (EERP/USP). Bolsista do Projeto de Extensão Universitária: Núcleo de Estudos e Recursos da Família.

2 Professora Doutora do Departamento de Enfermagem Psiquiátrica e Ciências Humanas da EERP/USP. sugalera@eerp.usp.br
\end{abstract}

\begin{abstract}
RESUMO
A esquizofrenia é um dos principais problemas de saúde da atualidade, exigindo considerável investimento do sistema de saúde. A intervenção no primeiro episódio do transtorno oferece uma oportunidade única no tratamento da esquizofrenia, influenciando no curso da doença. $O$ presente artigo consiste em uma revisão crítica de literatura cujos objetivos são examinar o conhecimento sobre o primeiro surto de esquizofrenia e discutir a contribuição da enfermagem na assistência. Foi utilizada pesquisa bibliográfica em índice informatizado de referências. Os dados obtidos permitiram organizar informações sobre o conceito geral de esquizofrenia, seu primeiro surto, tipos de intervenções e a atuação de enfermagem. Observamos que existe pouca literatura brasileira relacionada ao primeiro surto esquizofrênico, na área da enfermagem, poucos serviços especializados e disponíveis e poucos recursos

\section{ABSTRACT}

Schizophrenia is one of the main health problems in current days, requiring considerable investment from the health system. Intervening in the first episode offers a unique opportunity in the treatment of schizophrenia and influences the course of the illness. This article consists of a critical literature review aimed at examining knowledge on first episode schizophrenia and discussing the contribution of nursing care. A research was carried out in bibliographical databases. The data collected made possible the organization of information on the general concept of schizophrenia, its first episode, types of intervention and nursing performance. We found out that in Brazil there are few studies related to first episode schizophrenia in Nursing, few available specialized services, and few social resources. This situation reveals the need for more studies on first episode schizophrenia.
\end{abstract} sociais. Tal condição mostra a necessidade de estudos relacionados ao primeiro surto.

\section{DESCRITORES}

Esquizofrenia.

Enfermagem psiquiátrica.

Doença crônica.

\section{KEY WORDS}

Schizophrenia.

Psychiatric nursing.

Chronic disease.

\section{RESUMEN}

La esquizofrenia es uno de los principales problemas de salud de la actualidad, exigiendo considerable inversión del sistema de salud. La intervención en el primer episodio del trastorno ofrece oportunidad única en el tratamiento de la esquizofrenia, influyendo en el curso de la enfermedad. El presente artículo consiste en una revisión crítica de la literatura cuyos objetivos son examinar el conocimiento sobre el primer episodio de esquizofrenia y discutir acerca de la contribución de la enfermería en la asistencia. Fue utilizada la investigación bibliográfica en índice informatizado de referencias. Los datos obtenidos permitieron organizar informaciones sobre el concepto general de esquizofrenia, su primer episodio, tipos de intervenciones y la actuación de enfermería. Observamos que existe poca literatura brasileña relacionada a la primera aparición de signos de esquizofrenia, en el área de la enfermería, pocos servicios especializados disponibles y pocos recursos sociales. Tal condición muestra la necesidad de estudios relacionados al primer episodio.

\section{DESCRIPTORES}

Esquizofrenia.

Enfermería psiquiátrica. Enfermedad crónica. 


\section{INTRODUÇÃO}

A esquizofrenia é um dos principais problemas de saúde pública da atualidade, exigindo considerável investimento do sistema de saúde e causando grande sofrimento para o doente e sua família. Apesar da baixa incidência, por ser uma doença de longa duração, acumula-se, ao longo dos anos, um número considerável de pessoas portadoras desse transtorno, com diferentes graus de comprometimento e de necessidades.

A intervenção no primeiro episódio do transtorno oferece uma oportunidade única no tratamento da esquizofrenia. Sabe-se que a demora na procura do tratamento tem uma influência fundamental no prognóstico do paciente, pois pode levar a uma ruptura significativa dos níveis psíquico, físico e da rede social do doente. O tempo de tratamento para obtenção da remissão do quadro agudo também aumenta à medida que se sucedem os episódios psicóticos ${ }^{(1)}$.

Dada a importância do transtorno psicótico, tanto para a saúde pública como para o indivíduo e sua família, a finalidade deste artigo é a revisão de literatura sobre o primeiro surto da esquizofrenia e a importância das ações de enfermagem neste momento. $\mathrm{O}$ estudo de revisão de literatura tem grande importância para a formação e apresentação do conhecimento em todas as áreas. Na formação do aluno de graduação em enfermagem, a revisão de literatura permite o aprendizado sobre busca e leitura de textos na literatura científica e de como organizá-la para obter suporte teórico que oriente a prática da profissão, no sentido do cuidado eficaz e de qualidade ${ }^{(2)}$.

É importante descobrir quais intervenções podem contribuir no tratamento do primeiro surto esquizofrênico e como essas cumprem o papel de ajudar o indivíduo e sua família a se colocarem frente aos seus problemas e estigmas associados ao transtorno. Esta revisão deve, por conseqüência, refletir as vantagens da intervenção no primeiro surto e a contribuição da enfermagem nesse contexto. Dessa maneira, indicar como a enfermagem pode reduzir o sofrimento físico e emocional das pessoas que estão vivenciando o primeiro surto psicótico.

\section{OBJETIVOS}

Os objetivos desta revisão são:

- Examinar o conhecimento sobre a esquizofrenia e o primeiro surto em esquizofrenia.

- Examinar o conhecimento sobre a intervenção no primeiro surto de esquizofrenia e sua efícácia.

- Examinar o conhecimento da enfermagem sobre o primeiro surto em esquizofrenia, destacando a contribuição da profissão nesta área.

\section{MÉTODO}

Trata-se de um estudo bibliográfico, através de livrotexto e levantamento de artigos científicos na base de dados: MEDLINE, CAPES, LILACS e DEDALUS. Para a localização dos artigos foram usadas as seguintes palavras-chaves: esquizofrenia, primeiro episódio esquizofrênico, primeiro surto de esquizofrenia, enfermagem e esquizofrenia, família de esquizofrênico e os mesmos termos em inglês. Foram incluídos os artigos dos últimos quinze anos. A técnica utilizada foi a análise da bibliografia encontrada, que compreende a leitura, seleção, fichamento e arquivo dos tópicos de interesse para a pesquisa em pauta. Foram encontrados dezessete artigos e uma tese de interesse para este estudo, os quais enfocam evolução histórica do conceito de esquizofrenia, curso e prognóstico, primeiro episódio e seu início, atuação da enfermagem e tipos de intervenções os quais são apresentados no próximo item deste trabalho.

\section{DESENVOLVIMENTO}

\section{Esquizofrenia}

A esquizofrenia é um transtorno causado por diversos fatores biopsicossociais que interagem, criando situações, as quais podem ser favoráveis ou não ao aparecimento do transtorno. Os fatores biológicos seriam aqueles ligados à genética e/ou aqueles que são devidos a uma lesão ou anormalidade de estruturas cerebrais e deficiência em neurotransmissores. Os fatores psicossociais são aqueles ligados ao indivíduo, do ponto de vista psicológico e de sua interação com o seu ambiente social, tais como: ansiedade muito intensa, estado de estresse elevado, fobia social e situações sociais e emocionais intensas. Enfim, indivíduos com predisposição podem desenvolver a doença quando estimulados por fatores biológicos, ambientais ou emocionais ${ }^{(3-4)}$.

A própria definição fala que é um transtorno de longa duração, durante o qual a pessoa experimenta períodos de crises e remissões que resultam em deterioração do funcionamento do doente e da família, causando diversos danos/ perdas nas habilidades de todo o grupo. Por exemplo, diminuição da habilidade para cuidar de si mesmo, para trabalhar, para se relacionar individual e socialmente e para manter pensamentos completos ${ }^{(5)}$.

Pessoas esquizofrênicas têm como característica a perda de associações de idéias, alucinações, afeto embotado, riso imotivado ou inapropriado, avoliação, alogia, delírios proeminentes, deterioração global do funcionamento, associações frouxas, desorganização da sintaxe, pensamento ilógico e, principalmente, pobre em acontecimentos ${ }^{(3,5-7)}$.

Não existe um curso típico da esquizofrenia devido à grande sintomatologia apresentada, que varia de indivíduo 
para indivíduo. Somente 5\% dos pacientes apresentam um surto na vida, e a maioria experimenta vários surtos, principalmente no início da doença. A variabilidade da evolução encontrada independe dos sintomas apresentados no início da doença.

\section{Idade de início}

A idade de início é tradicionalmente considerada como um fator importante para o prognóstico. Quando a doença se inicia antes dos 20 anos, o prognóstico é pior. A idade de início no homem é menor que na mulher, 15 a 25 anos e 25 a 35 anos respectivamente. Esta diferença é explicada na literatura com a seguinte argumentação: os rapazes sofrem estresse mais cedo que as moças, que apresentam taxas de hormônios contínuas. Os hormônios femininos têm efeitos parecidos com os neurolépticos, por isso os sintomas aparecem mais tardiamente, somente quando as taxas hormonais começam a diminuir ${ }^{(5)}$.

O final da adolescência e início da vida adulta é uma fase bastante conturbada, pois envolve transformações físicas, emocionais e aquisição de novas responsabilidades e papéis, da pessoa, em seu ambiente social. O aparecimento da psicose nesta época se dá, muitas vezes, pela pressão que o jovem sofre, dessas mudanças $^{(5)}$.

Hoje se sabe que a doença e a deterioração iniciam-se anos antes das manifestações clínicas mais características da psicose. A fase prodrômica se caracteriza por ansiedade, insônia, dificuldade de atenção e concentração, irritabilidade, deterioração da performance e das atividades cotidianas, distúrbios do comportamento, distanciamento social, comportamento desorganizado, paranóides alucinatórios, delírios menos freqüentes que alucinações. Os conteúdos dos delírios e alucinações variam de acordo com a idade de início da doença, nos mais jovens apresentam-se mais simples e fixos e nos mais velhos mais complexos ${ }^{(1,8)}$. Muitas vezes as pessoas que convivem com o doente atribuem às mudanças observadas nessa fase como típicas da adolescência e ou de uma crise da idade adulta e por isso costumam não procurar ajuda especializada. Geralmente a busca por ajuda ocorre somente quando os sintomas agudos aparecem.

\section{Formas de início}

A esquizofrenia pode apresentar início agudo ou o insidioso, os quais possuem características distintas evoluindo para uma sintomatologia própria. O início agudo é caracterizado pelo aparecimento dos sintomas de forma abrupta, evoluindo para uma deterioração se não tratados imediatamente. Tem como principal sintomatologia a regressão, a confusão e a ansiedade que progridem para estado de pânico, episódios confusionais do delírio febril, excitação motora, insônia e atitudes catatônicas ${ }^{(7-8)}$.
O início insidioso caracteriza-se por um desenvolvimento da psicose de forma mais amena, o desenvolvimento psicomotor é normal, porém nos aspectos emocional e intelectual manifestam-se desvios extremos. Aparecem fenômenos dissociativos nas áreas da afetividade e da linguagem, fortes mudanças de humor, atos obsessivos e compulsivos, irritabilidade e hostilidade. Quando não ocorre intervenção neste tipo de início, ele evolui para a deterioração do funcionamento global e exacerbação aguda da psicose em um próximo momento $^{(7-8)}$.

\section{Tratamento}

Idealmente $o$ atendimento de pacientes que estão vivendo o primeiro surto deveria se dar em locais especializados, como é o exemplo de um serviço existente em Ontário, Canadá, descrito em pesquisa ${ }^{(9)}$. No Canadá, é preconizada a presença de um serviço especializado no atendimento do primeiro surto psicótico para cada 390.000 habitantes.

Existe um intervalo de tempo entre o surgimento dos sintomas e a procura pelo tratamento. Este tempo pode influenciar no prognóstico. A demora na procura do tratamento pode indicar um mau prognóstico, pois os sintomas tornam-se mais intensos, implicando em maior tempo de tratamento psicofarmacológico e com doses mais elevadas. Por isso é recomendada a criação de serviços em saúde mental destinados ao atendimento de adolescentes e jovens, os quais poderiam contribuir para detectar e tratar precocemente o primeiro surto da esquizofrenia.

A intervenção adequada envolve o tratamento farmacológico, psicossocial e a inclusão da família. Deve-se fazer um diagnóstico diferenciado de cada paciente, respeitando sua individualidade ${ }^{(9)}$. A avaliação e a assistência devem ser feitas por uma equipe multiprofissional, composta no mínimo de médico psiquiatra, terapeuta ocupacional, enfermeira com especialização em psiquiatria e assistente social ${ }^{(1,9-10)}$. A internação psiquiátrica deve ser evitada, dando-se preferência para tratamento intensivo na comunidade, durante a fase aguda, e seguimento nos dois anos seguintes com o objetivo de se prevenirem recaídas e contribuir na adaptação do doente e sua família nesse período considerado crítico $^{(11)}$.

\section{Tratamento Farmacológico}

O tratamento farmacológico no primeiro episódio da esquizofrenia consiste no uso de medicamentos antipsicóticos, chamados de neurolépticos. Existem dois tipos de drogas antipsicóticas: os antipsicóticos típicos ou convencionais e os atípicos.

Os antipsicóticos típicos ou convencionais são antagonistas da dopamina e seu efeito resulta na diminuição dos 
sintomas positivos (delírios, alucinações, pensamento incoerente) e na produção de efeitos colaterais, principalmente os efeitos extrapiramidais, que têm como sintomas básicos transtornos dos movimentos como parkinsonismo (tremor, rigidez e bradicinesia), distonia e acatisia. O uso prolongado e inadequado dos antipsicóticos típicos contribui para uma disfunção crônica e irreversível chamada discinesia tardia, que também é um efeito colateral extrapiramidal. Os efeitos colaterais desse grupo de medicamentos são um dos principais fatores que contribuem para a não-adesão ao tratamento psicofarmacológico ${ }^{(12-13)}$. Os principais medicamentos usados em nosso meio são a Clorpromazina e o Haloperidol.

Os antipsicóticos atípicos ou recentes inibem receptores de dopamina e serotonina, melhorando sintomas positivos e ajudando no tratamento de sintomas negativos sem efeitos extrapiramidais significativos. $\mathrm{O}$ aumento de peso é o efeito colateral mais significativo desse grupo de medicamentos e deve ser acompanhado. Os principais medicamentos são a Clozapina, Risperidone e o Olanzapine Os antipsicóticos de nova geração vêm substituindo os demais, no tratamento do primeiro episódio esquizofrênico, devido à possibilidade do uso de doses mais baixas e, conseqüentemente, menores efeitos colaterais $^{(13-14)}$.

A clozapina é o único antipsicótico de nova geração que não é recomendado para o início do tratamento, pois ele tem efeitos colaterais mais agravantes. $\mathrm{O}$ efeito adverso mais sério é a agranulocitose, que exige exames laboratoriais freqüentes e precisa de um monitoramento semanal ${ }^{(13-14)}$.

A tolerância de resposta a esses medicamentos deve ser de 6 a 8 semanas após o início do uso. A não-resposta ao primeiro medicamento em uso implica na substituição por outro, e isso deve ocorrer até se encontrar a droga mais adequada ao paciente de acordo com suas características ${ }^{(1,3)}$.

Doses altas e intensivas são aplicadas em episódios agudos e durante crises de recaída, devendo diminuir com a melhora do quadro, para que a crise seja controlada e o quadro psicótico mantenha-se em equilíbrio. Dessa maneira diminui-se o efeito colateral e melhora-se a qualidade de vida do doente e de sua família que se sentirão mais seguros e confiantes no tratamento ${ }^{(1,3)}$.

O tratamento farmacológico deve ser mantido, pelo menos, durante os dois primeiros anos, após o primeiro surto, para controlar uma recaída mais violenta ${ }^{(1)}$. Quando o paciente apresenta fobia social e ataques/transtornos de pânico, o tratamento farmacológico pode ser mantido com o objetivo de aliviar os $\operatorname{sintomas}^{(3)}$. O paciente e a família devem ser orientados sobre os perigos do uso/abuso de substâncias não prescritas e de altas taxas da medicação prescrita sem recomendação médica, para não debilitá-lo ainda mais ${ }^{(9)}$.

\section{Intervenção Psicossocial}

A intervenção psicossocial consiste no tratamento do paciente, baseado no envolvimento deste com atividades sociais e ocupacionais. Ela está sendo utilizada para diminuir o estigma da doença mental perante a sociedade e ao próprio usuário que, muitas vezes, sente-se inibido ao procurar ajuda, acarretando em uma deterioração que poderia ser evitada ${ }^{(10)}$.

A intervenção psicossocial vem se desenvolvendo como um campo de estudo próprio, sendo o conceito de reabilitação psicossocial uma de suas bases. Por ser muito nova e abrangente, observa-se que existem muitas práticas distintas que utilizam o termo, por isso é importante adotar uma definição de reabilitação psicossocial que fundamente nossa prática ${ }^{(15)}$.

Estudos mostram que o atendimento domiciliar, quando o episódio agudo está controlado, é a melhor alternativa ${ }^{(1)}$. Além do engajamento familiar com o paciente, o qual será abordado mais adiante no texto, este tipo de intervenção encoraja a busca de recreação, educação, serviços vocacionais disponíveis na comunidade e programas de reabilitação $0^{(9,16-17)}$.

Outra característica da intervenção psicossocial é que ela busca a recuperação e aceitação da doença, a quebra de estigma, através da participação em atividades grupais, com direção orientada $^{(10)}$. Através dos grupos, há um período de avaliação, culminando na oportunidade para o mútuo apoio durante a transição da recuperação dos sintomas agudos psicóticos, promovendo um conceito individual dos pacientes sobre a doença e proporcionando uma melhor adaptação da psicose. Conseqüentemente, um melhor conforto ao paciente ${ }^{(10)}$.

O seguimento em serviços de reabilitação social para pacientes mais velhos e com maior comprometimento não é recomendado para pacientes do primeiro surto, porque pode influenciar negativamente o jovem na sua esperança e empenho em cuidar de sua saúde mental. $\mathrm{O}$ uso de recursos da comunidade para jovens saudáveis pode ser mais adequado ${ }^{(9)}$.

\section{Intervenção Familiar}

A intervenção familiar vem sendo uma alternativa indispensável no primeiro episódio esquizofrênico. Deve-se ter um conhecimento, primeiramente, da família, suas características, limitações, medos e inseguranças. Sabe-se que, no momento em que a família se depara com a nova situação, ocorre uma desorganização do grupo na tentativa de se adap$\operatorname{tar}^{(18)}$. Se a família não for ajudada neste momento, sua adaptação pode resultar em modelos de relacionamento que contribuem pouco para a estabilização e melhora do paciente e, leva todo o grupo ao sofrimento mental. Uma adaptação mais positiva pode ser de grande valor para a recuperação e inclusão do doente mental e para a qualidade de vida de todo o grupo ${ }^{(18)}$. 
A intervenção familiar do tipo sistêmico é a mais recomendada nesse período. Através dela é possível promover educação sobre a doença, seus sintomas, suas crises, seu tratamento, suas delimitações, além de ajudar o grupo a enfrentar melhor o impacto da doença, promovendo também a redução do estigma ${ }^{(9,16-18)}$.

A intervenção de tipo sistêmica não deve durar os dois anos. É recomendável um trabalho inicial de no mínimo cinco encontros, com intervalo de 15 dias e, em seguida, encontros mais esporádicos, pois é preciso dar autonomia para a família de modo que, ela se sinta capaz de se autocuidar ${ }^{(19)}$. Os grupos de familiares podem durar mais tempo.

Infelizmente a família ainda é considerada como mero informante dos sintomas do doente, sendo pouco considerado o seu sofrimento. O ideal é acolher a família promovendo suporte possível para as demandas manifestadas tais como culpa, conflitos, situações de crise, isolamento social, etc. Os serviços de saúde devem se estruturar para potencializar a relação familiar/profisssional/serviço ${ }^{(20)}$.

\section{O cuidado de Enfermagem}

A enfermagem psiquiátrica está fundamentada no relacionamento interpessoal enfermeira-paciente, através do qual observa os aspectos biopsicossociais do ser humano. No aspecto biológico, a enfermagem observa efeitos colaterais da medicação e acompanha a saúde geral do jovem paciente e de sua família. No campo psicossocial, pode se envolver em diversas atividades, tais como a visita domiciliária, a coordenação de grupos de pacientes em oficinas e outros temas. A promoção do acesso do paciente e família aos recursos da comunidade pode contribuir para a reabilitação do doente e da família. O cuidado de enfermagem, com enfoque no sistema familiar, tem se mostrado bastante útil por permitir observar os aspectos biopsicossociais do paciente e de sua família e contribuir para uma melhor articulação do grupo com a comunidade ${ }^{(19)}$.

A avaliação das necessidades específicas e as ações de enfermagem são aplicadas de acordo com a individualidade de cada família. Assim, têm-se uma reorganização dos sintomas dos pacientes e uma prevenção para futuros episódios, melhorando a qualidade de vida do grupo familiar, seu papel frente à sociedade e entre seus próprios membros, evitando a deterioração definitiva que leva à incapacidade mental.

As ações de enfermagem ocorrem em serviços específicos para o atendimento do primeiro surto e em serviços de saúde primária. Assim que pacientes e famílias apresentam uma melhor aceitação da nova condição, são encorajados a enfrentá-la através de atividades com recursos da comunidade, o que possibilita a recuperação da vida social e, uma reabilitação mais rápida e eficiente.

A prática em enfermagem psiquiátrica se baseia em ações que visam a melhorar a condição da qualidade de vida do paciente e de sua família, a contribuir no controle do surto da doença, torná-la estabilizada, a ajudar na integração social após o aparecimento da doença, e a cooperar na adesão ao tratamento e à adaptação de sua nova condição.

As ações de enfermagem discutidas na literatura são: implementar avaliações biopsicossociais com atenção às características culturais do paciente; criar e implementar planos para melhorar as condições de saúde do paciente e de sua família; orientar paciente e família sobre as características da doença, do tratamento e sobre os recursos disponíveis; promover e manejar, dentro da saúde mental, os efeitos da doença através do ensino, da pesquisa, proporcionando adequado aconselhamento à família e ao paciente; manejar e coordenar sistemas de integração de cuidados que integrem as necessidades do paciente e da família, promovendo um entendimento e uma melhor aceitação da doença, o que leva à melhor adesão ao tratamento e uma melhor reabilitação social ${ }^{(13)}$. Outra importante ação da enfermagem é a estimulação dos pacientes de primeiro surto esquizofrênico a usar recursos disponíveis na sociedade como trabalhos voluntários, atividades em grupos, exercícios físicos, lazer, entre outros.

\section{CONSIDERAÇÕES FINAIS}

Com a presente revisão e leitura críticas dos textos científicos, principalmente sobre enfermagem, observamos que ainda existe pouca literatura sobre o tema. Encontramos na literatura mais informações sobre o que se deve ou se pode fazer. Estas informações são bastante úteis aos estudantes e profissionais de enfermagem, pois permite que eles organizem suas ações. Porém, sabemos muito pouco sobre a eficácia das intervenções e sobre as condições em que elas podem ser utilizadas. Poucos trabalhos descrevem problemas vividos pelo paciente e sua família e as intervenções em enfermagem que ajudaram o grupo a resolvê-los ${ }^{(19)}$. Dessa maneira, consideramos que esta é uma área na qual a enfermagem pode e deve desenvolver pesquisa, oferecendo à comunidade científica e profissional um rol de problemas e ações de enfermagem e de suas contribuições para melhorar a qualidade de vida do paciente e da sua família, também conhecimentos sobre o transtorno mental e as ações de enfermagem que possibilitem nosso desenvolvimento teórico.

$\begin{aligned} \text { Rev Esc Enferm USP } & \text { Primeiro episódio da esquizofrenia e } \\ 2006 ; 40(2): 286-91 . & \text { assistência de enfermagem } \\ \text { www.ee.usp.br/reeusp/ } & \text { Giacon BCC, Galera SAF. }\end{aligned}$




\section{REFERÊNCIAS}

(1) Louzã Neto MR. Manejo clínico do primeiro episódio psicótico. Rev Bras Psiquiatr. 2000;22(suppl 1):45-6.

(2) Stevens KR, Long JD. Incorporating systematic reviews into nursing education. Online J Knowl Synth Nurs. 17(5) [Serial online]. Available from: http://www.stti.org/VirginiaHenderson Library/PDFs/ec_doc1e.pdf [Access at 17 nov. 1998].

(3) Figueira I, Mendlowicz M, Nardi AE, Marques C, Ventura P, Moraes A, et al. Fobia social e transtorno do pânico em pacientes psicóticos. J Bras Psiquiatr. 1992;41(6):259-64.

(4) Lima COM, Caetano D. Evolução histórica do conceito de esquizofrenia. J Bras Psiquiatr. 1995;44(11):559-62.

(6) Tostes LRM, Moraes LRN. Esquizofrenia: curso, evolução e prognóstico. J Bras Psiquiatr. 1989;38(4):233-9.

(6) Amaral M, Cheniaux Jr. E. Psicoses com características afetivas e esquizofreniformes simultâneas. J Bras Psiquiatr. 1992;41 (6):297-302.

(7) Ferrari MCL. Esquizofrenia na infância e adolescência: um esboço histórico. Rev Psiquiatr Clin. 1996;23(3):97-104.

(8) Alvarenga-Silva H, Teixeira Jr AL, Costa C. Esquizofrenia de início na infância: uma revisão da apresentação clínica e da evolução. J Bras Psiquiatr. 2000;10(12):387-93.

(9) Malla AK, Norman RMG, Terry SM, Mclntosh E. Impact of phase specific treatment of first episode of psychosis Wisconsen Quality of Life Index (client version). Acta Psychiatr Scand. 2001;103:355-61.

(10) Maccay E, Ryan K, Amey S. Mitiganting Engulfment: recovering from a first episode of psychosis. J Psychosoc Nurs. 1996;34(11):40-4.

(11) Menezes PR. Prognóstico da esquizofrenia. Rev Bras Psiquiatr. 2000;22(Suppl 1):18-20.
(12) Abreu PB, Bolognesi G, Rocha N. Prevenção e tratamento de efeitos adversos de antipsicóticos. Rev Bras Psiquiatr. 2000;22(Suppl 1):44-8.

(13) Kaplan HI, Sadock BJ, Grebb JA, editores. Compêndio de psiquiatria: ciências do comportamento e psiquiatria clínica. $7^{\mathrm{a}}$ ed. Porto Alegre: Artes Médicas; 1997. Terapias biológicas. p. 809-946.

(14) Laraia MT. Psicofarmacologia. In: Stuart GW, Laraia MT, editores. Enfermagem psiquiátrica: princípios e prática. $6^{\mathrm{a}} \mathrm{ed}$. Porto Alegre: Artmed; 2001. p. 607-39.

(15) Kirschbaum DIR. O trabalho de enfermagem e o cuidado em saúde mental: novos rumos? Cad IPUB. 2000;(19):15-36.

(16) Czuchta DM, Maccay E. Help-Seeking for parents of individuals experiencing a first episode of schizophrenia. Arch Psychiatr Nurs. 2001;15(4):159-70.

(17) Coldham EL, Addington J, Addington D. Medication adherence of individuals whit a first episode of psychosis. Acta Psychiatr Scand. 2002;106(4):286-90.

(18) Schneider JF. O cuidado e a família do esquizofrênico. Mundo Saúde. 2000;24(24):284-90.

(19) Galera SAF. Avaliação construtiva de uma intervenção de enfermagem junto a famílias que tem um portador de esquizofrenia entre seus membros [tese]. Ribeirão Preto: Escola de Enfermagem de Ribeirão Preto/USP; 2002.

(20) Colvero LA, Ide CAC, Rolim MA. Família e doença mental: a difícil convivência com a diferença. Rev Esc Enferm USP. 2004;38(2):197-205. 